What is already known on this topic

It is estimated that a Snellen acuity of $6 / 9^{-2}$ produces the same failure rate as the number plate test at $20.5 \mathrm{~m}$, leading to guidelines that a visual acuity between $6 / 9$ and $6 / 12$ is required for driving

\section{What this study adds}

A proportion of patients with binocular $6 / 9$ vision failed to read a number plate at $20.5 \mathrm{~m}$, and a major proportion with binocular $6 / 12$ vision could read it

Snellen visual acuity is a poor indicator of an individual's chance of meeting the legal standard for driving, and all people with a visual acuity of $6 / 9$ or less should be encouraged to self assess their vision

Patients receive a spectrum of advice from healthcare professionals about driving on the basis of a visual acuity of $6 / 9$ or $6 / 12$

distance outside the practice-this would provide a useful public service.

\section{Conclusions}

Snellen acuity is a poor indicator of an individual's chance of passing a number plate test, and it cannot be assumed that a driver with a visual acuity of $6 / 9$ will meet the standard for driving. All drivers with 6/9 vision or less must be encouraged to self assess. Conversely, it should not be assumed that a driver with a visual acuity of $6 / 12$ is below the standard for driving, particularly as loss of the right to drive has such far reaching social implications. ${ }^{78}$ Advice that vision required for driving lies somewhere between $6 / 9$ and $6 / 12$ acuity could cause confusion if these points are not stressed.

AB's current address is Queens Medical Centre, Nottingham NG7 2UH.

Contributors: $\mathrm{ZC}$ contributed to the design of the study and questionnaire, distribution of the questionnaire, patient testing, analysis of the results, and writing the paper; she will act as guarantor. $\mathrm{AB}$ contributed to the distribution of the questionnaire, patient testing, and writing the paper. IP contributed to the analysis of the results, patient testing, and writing the paper.

Funding: None

Competing interests: None declared.

1 Driver and Vehicle Licensing Authority. At a glance guide to the current medical standards of fitness to drive. Swansea: Drivers Medical Unit, DVLA, 1998:25-6.

2 Marsden AM, Packer AL. The visibility of vehicle number plates. Trans Illuminating Eng Soc (London) 1966;31:2.

3 Drasdo N, Haggerty CM. A comparison of the British number plate and Snellen vision tests for car drivers. Ophthalmol Physiol Opt 1981;1:39-54.

4 Taylor SP. Accuracy of recall of the legal number plate testing distance by UK drivers. Ophthalmol Physiol Opt 1997;17:473-7.

5 Department of Transport. Consumer trends survey Aug, 1998

6 McCaghrey GE. AOP vision screening 1985/86. Optom Today 1987;27:178-81.

7 Shipp MD, Penchanski R. Vision testing and the elderly driver: is there a problem meriting policy change? J Am Optom Assoc 1995;66:343-51.

8 Charman WN. Visual standards for driving. Ophthalmol Physiol Opt 1985;5:211-20.

(Accepted 24 July 2000)

\title{
Unjustified exclusion of elderly people from studies submitted to research ethics committee for approval: descriptive study
}

Antony Bayer, Win Tadd

Correspondence to: A Bayer

bayer@cf.ac.uk

continued over

BMJ 2000;321:992-3

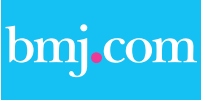

This article is part of the BMJ's randomised controlled trial of open peer review. Documentation relating to the editorial decision making process is available on the BMJ's website
Ageism in clinical practice ${ }^{1}$ and published research ${ }^{2}$ is well recognised. We were interested in whether research protocols submitted to the local research ethics committee contained unjustified upper age limits and how the committee dealt with this.

\section{Methods and results}

We reviewed all studies submitted to Bro Taf local research ethics committee in the first seven months of 1999 to determine whether any upper age limits were justified and whether the committee had commented on such age restrictions. We then made a judgment on the appropriateness of the upper age limit.

Of 225 studies whose protocols were reviewed, 65 were on topics or conditions that automatically excluded elderly people. Five studies specifically concerned elderly people and had a lower age limit but no upper limit. Of the remaining $155,90(58 \%)$ had an upper age limit, which ranged from 45 years (in a smoking cessation intervention) to 100 years (in a study of an open access mental health service), with a median of 70 years (interquartile range 65 to 75 years). In five studies an upper age limit was reasonable (participants were required to have no important disease, or prolonged follow up was planned).

In 85 studies the age restriction was inappropriate and unnecessary, but ethical review had failed to highlight this issue. Justification was offered by researchers in only one study. Age limits often conflicted with the aims of the study-for example, in a study of "subjects randomly selected to reflect each life stage," another "of consecutive patients attending for bone scan," and another that claimed "no special groups to be excluded." In some studies exclusion of older patients was likely to result in an atypical clinical populationfor example, an investigation of "exclusion of vulnerable people from services," studies of type II diabetes, glaucoma, or non-steroidal anti-inflammatory drugs that were restricted to people aged under 65 , and a study comparing incontinence aids that was limited to those aged under 70 . 
A total of 46 studies were approved by a multicentre research ethics committee; of these 20 had an upper age limit that seemed unjustifiable.

\section{Comment}

Of the 155 studies that were of relevance to elderly people, over half had an upper age limit that was unjustified, and neither the local ethics committee nor the multicentre research ethics committee had requested justification for the age restrictions.

Negative stereotyping of elderly people was reflected in comments in the studies that participants need to be "fully competent," "reliable," or "without cognitive impairment." One argument for an upper age limit is that it minimises the rate of dropout. However, we know of no evidence that elderly people are less compliant with the demands of research protocols, and their fewer family and employment commitments may make participation easier.

Furthermore, exclusion of elderly participants can affect the generalisability of a study's findings. If researchers can be certain that elderly people will not respond differently from other age groups, their specific inclusion may not be an issue; if this is not the case, however, their inclusion is essential. ${ }^{3}$ Abolishing ageist practices and attitudes in research, as well as in clinical practice, is important if elderly people are to gain maximum benefit from advances in health care. ${ }^{4}$

Ethics committees are in a strong position to influence research practice and to reduce unethical age discrimination. We encourage them to request justification whenever protocols include inappropriate age restrictions-and if this is not forthcoming, approval might be conditional on age limits being removed. This policy would promote more positive attitudes towards elderly people among researchers as well as safer, more effective treatments and services.

We thank Carl Phillips (executive officer, Bro Taf local research ethics committee) for his administrative help and support.

Contributors: $\mathrm{AB}$ and WT designed and performed the study and wrote the paper. $\mathrm{AB}$ is guarantor for the paper.

Funding: None.

Competing interests: $\mathrm{AB}$ and $\mathrm{WT}$ are both members of Bro Taf local research ethics committee.

1 Bowling A. Ageism in cardiology. BMJ 1999;319:1353-5.

2 Bugeja G, Kumar A, Banerjee AK. Exclusion of elderly people from clinical research: a descriptive study of published reports. BMJ 1997;315:1059.

3 European Commission Committee on Proprietary Medicinal Products. Guidelines on clinical investigation of medicinal products in the elderly. Brussels: CPMP, 1998

4 Age Concern. Turning your back on us. London: Age Concern, 1999.
University Department of Geriatric Medicine, University of Wales College of Medicine, Llandough Hospital, Penarth CF64 2XX

Antony Bayer director, Cardiff

Memory Team Win Tadd research fellow

(Accepted 16 June 2000)

\section{A memorable patient \\ Never has a three hour flight seemed so long}

"The pilot would like to know if you would have him land the airplane in Bermuda," the British West Indies Airline flight attendant asked, quietly and professionally. "No," I replied, "there is no cardiac surgeon there."

Reisha, my 4 month old patient, had picked an unfortunate day to try to die. We knew that she had heart disease soon after she was born. She first developed cyanotic spells, the hallmark of tetralogy of Fallot, at 2 months. In Antigua and Barbuda it takes time to arrange for overseas evaluation and care, but with the help of the Rotary club and its Gift of Life programme we had been able to arrange for transport to Schneider Children's Hospital in New York.

In the three days before departure the cyanotic spells became more frequent and more pronounced. She had been treated with oxygen, morphine, and propranolol. An air ambulance would have been more appropriate for transfer, but the cost is overwhelming in a developing country. So we claimed that she was fit to fly on a commercial flight. A nurse from the special care unit at Holberton Hospital accompanied Reisha, her mother, and me.

Reisha dropped her oxygen saturation to $40 \%$ in the ambulance on the way to the airport, so we drove on to the tarmac and boarded while providing her with bag and mask ventilation with oxygen. She did not seem to like the rarefied atmosphere over the Atlantic and proceeded to drop her oxygen level to $30 \%$ on a regular basis. She was given morphine and needed ventilation for her subsequent apnoea and to try to lower her pulmonary resistance to improve lung blood flow. Her oxygen rose temporarily to $95 \%$ and then it would fall again.

We had adrenaline, which we began as a bolus to raise systemic resistance and theoretically increase the blood flow to the lungs. It worked when the oxygen level fell to $40 \%$, but after each treatment Reisha's pulse and oxygen level were not detectable for about five minutes. My heart would stop and restart when her oxygen level rose again to $95 \%$. We began chest compressions when we weren't sure. Between adrenaline doses the intravenous line dislodged. I didn't need to tell the nurse that failure to resite the line would result in Reisha's death. The nurse replaced the line at the first try, in a darkened airplane, at 30000 feet.

It was then that the flight attendant made her offer. About that time the adrenaline bolus technique was becoming less effective, so we began an adrenaline infusion and occasional sodium bicarbonate. Mainlining adrenaline is a desperate measure, even at sea level.

I don't think that a three hour flight ever lasted so long. But, together with bag and mask oxygen, adrenaline, bicarbonate, and morphine, we made it to New York. We were processed in our seats and the transport team worked for 45 minutes before Reisha was stable enough for the ambulance drive. On arrival at the intensive care unit her $\mathrm{paO}_{2}$ was 25 torr and her $\mathrm{pH}$ 7.40. I worried about her brain.

She underwent emergency placement of a Blaylock-Taussig shunt and complete repair of her tetralogy of Fallot defect one year later. She is now 4 and due to start school this fall with her twin sister and is now developing normally.

Thomas C Martin consultant paediatrician and cardiologist, Antigua

We welcome articles of up to 600 words on topics such as A memorable patient, A paper that changed my practice, My most unfortunate mistake, or any other piece conveying instruction, pathos, or humour. If possible the article should be supplied on a disk. Permission is needed from the patient or a relative if an identifiable patient is referred to. We also welcome contributions for "Endpieces," consisting of quotations of up to 80 words (but most are considerably shorter) from any source, ancient or modern, which have appealed to the reader. 